\title{
Growth properties of solutions of second order elliptic differential equations
}

\author{
By
}

Kiyoshi MochIzukI

(Communicated by Prof. Mizohata, July 24, 1975)

\section{Introduction}

In this paper we consider the equation

$$
-\sum_{j, k=1}^{n} D_{j} a_{j k}(x) D_{k} u-q(x) u+p(x) u=0
$$

in an exterior domain $\Omega \subset \mathbf{R}^{n}$, where $D_{j}=\partial_{j}+i b_{j}(x)$ with $\partial_{j}=\partial / \partial x_{j}$ and $i=\sqrt{-1}$, and the matrix $\left(a_{j k}(x)\right)$ is uniformly positive definite in $x \in \Omega$ (the precise condition on the coefficients will be given later). We assume that $a_{j k}(x) \rightarrow \delta_{j k}$ (Kronecker's delta) as $|x| \rightarrow \infty$, that $\partial_{k} b_{j}(x)$ $-\partial_{j} b_{k}(x)$ and $p(x)$ behave like $o\left(|x|^{-1}\right)$ as $|x| \rightarrow \infty$ and that there exist some constants $0<\gamma_{0}<1, \lambda_{0}>0$ and $r_{0}>0$ such that the domain $B\left(r_{0}\right)$ $=\left\{x ;|x|>r_{0}\right\}$ is included in $\Omega$ and

(2) $\quad 2 \gamma_{0}\left(\sum_{j, k} a_{j k}(x) \tilde{x}_{j} \tilde{x}_{k}\right) q(x)+|x| \sum_{j, k} \tilde{x}_{j} a_{j k}(x) \partial_{k} q(x) \geq \lambda_{0}$ for $x \in B\left(r_{0}\right)$,

where $\tilde{x}=x /|x|$. The main purpose of the present paper is to derive a growth estimate at infinity of solutions $u(x)$ of equation (1), from which will follow the uniqueness of $L^{2}$-solutions of (1).

Equations of the form (1) appear frequently in applications. In particular, if we assume that $a_{j k}(x)=\delta_{j k}$ and $q(x)=\lambda-c(x)$, where $\lambda>0$, then (1) becomes

$$
-\sum_{j=1}^{n} D_{j}^{2} u+(c(x)+p(x)) u-\lambda u=0
$$


which is the eigenvalue problem of the Schrödinger operator (in this case $n=3 s, s$ being the number of particles), where $c(x)+p(x)$ represents the potentials of interaction between the particles. By the diminishing condition at infinity $\left(b_{j}(x)\right)$ and $p(x)$ represent especially the magnetic vector potentials and the short-range scalar potentials in the twobody problem, respectively. $q(x)=\lambda-c(x)$ satisfies inequality (2) with $0<\gamma_{0}<1,0<\lambda_{0} \leq 2 \gamma_{0} \lambda$ and $r_{0} \gg 0$ if $c(x)$ satisfies one of the following three conditions:

(c.1) $\quad c(x)=0$;

(c.2) $c(x)$ and $|x| \sum_{j} \tilde{x}_{j} \partial_{j} c(x)$ tend to zero as $|x| \rightarrow \infty$;

(c.3) $-2 \gamma_{0} c(x) \geq|x| \sum_{j} \tilde{x}_{j} \partial_{j} c(x)$ for $x \in B\left(r_{0}\right)$.

By (c.2) is given a class of long-range potentials in the two-body problem. (c.3) holds if $c(x)$ is a homogeneous function of degree $-2 \gamma_{0}$. Thus some potentials appearing in the manybody problem satisfy (c.3). Thus, from the physical point of view, our problem of the present paper may be said to show the non-existence of the positive eigenvalues of the Schrödinger operator.

In the case where $c(x)$ satisfies (c.1) or (c.2), growth properties of solutions, hence the uniqueness of $L^{2}$-solutions, of equation (3), or some of its variants, have been investigated by many authors ([1] $\sim[4],[6],[8],[10],[11])$. There are also several works ([1], [2], $[12] \sim[14]$ ) investigating the Schrödinger equation

$$
-\Delta u+c(x) u-\lambda u=0 \quad(\lambda>0) \quad \text { in } \Omega
$$

with $c(x)$ satisfying (c.3). Among them, Uchiyama [14] gave an explicit formulation of the asymptotic estimates at infinity of solutions of (4) assuming that $\gamma_{0}$ in (c.3) satisfies the condition $\frac{1}{3}<\gamma_{0} \leq 1$. He also proved, as a special problem, that if $\Omega=\mathbf{R}^{n}$ and (c.3) is satisfied for all $x \in \mathbf{R}^{n}$, then the condition on $\gamma_{0}$ can be weakened to the condition $0<\gamma_{0} \leq 1$.

We shall deal with the "exterior" problem with $\gamma_{0}$ satisfying the 
condition $0<\gamma_{0}<1$. Our results will not exclude the case of $\Omega=\mathbf{R}^{n}$. Since equation (1) is rather general, it seems difficult to develop a theory including the case of $\gamma_{0}=1$. However, as we shall show later, for the simpler equation (4) we can obtain a result including the case of $\gamma_{0}=1$ (Theorem 1.3).

Our method is very close to those developed by Roze [10] and Éidus [3] for equations with short-range potentials. We use two equalities which follow from equation (1). Roughly speaking, the first equality is a consequence of the integration by parts of (1) multiplied by $|x|^{\alpha} \bar{u}$ and the second equality is similarly given from (1) multiplied by $|x|^{\beta} \sum_{j, k} \tilde{x}_{j} a_{j k}(x) \overline{D_{k} u}$. Combining these equalities, we obtain some convenient apriori estimates. In our proof a difficulty occurs in the existence of singularities of $q(x)$ which may spread out to infinity. In order to remove the influence of the singularities, we use not only condition (2) but also the first equality which includes the term

$$
\int|x|^{\alpha}\left(\sum_{j, k} a_{j k}(x) D_{j} u \overline{D_{k} u}-q(x)|u|^{2}\right) d x
$$

One can find similar treatments also in Weidmann $[12,13]$, Agmon $[1,2]$ and Uchiyama [14].

\section{§1. Notation and Results}

First we shall list the notation which will be used freely in the sequel:

$<f \cdot g>=\sum_{j=1}^{n} f_{j} g_{j}$ for $f=\left(f_{1}, \ldots, f_{n}\right)$ and $g=\left(g_{1}, \ldots, g_{n}\right)$

$|f|=\left\langle f \cdot f>1 / 2\right.$ for $f \in \mathbf{C}^{n}$;

$[f]_{j}$ denotes the $j$-th component of $f \in \mathbf{C}^{n}$;

$x=\left(x_{1}, \ldots, x_{n}\right)$ is a position vector in $\mathbf{R}^{n}$;

$r=|x|$ and $\tilde{x}=x /|x|$;

$S(t)=\{x ;|x|=t\}$ for $t>0$;

$B(s, t)=\{x ; s<|x|<t\}$ for $0<s<t ;$

$B(t)=\{x ;|x|>t\}$ for $t>0$;

$\partial_{j}=\partial / \partial x_{j}, \operatorname{grad} f=\left(\partial_{1} f, \ldots, \partial_{n} f\right)$ for scalar functions $f$ and $\operatorname{div} g$ 
$=\partial_{1} g_{1}+\cdots+\partial_{n} g_{n}$ for vector functions $g$;

$D_{j}=\partial_{j}+i b_{j}(x)$ and $D=\left(D_{1}, \ldots, D_{n}\right)$, where $i=\sqrt{-1}$;

$A=\left(a_{j k}(x)\right)$ and $A_{l}^{\prime}=\left(\partial_{l} a_{j k}(x)\right)(j, k=1, \ldots, n)$;

$B_{j}^{\prime}=\left(b_{j k}^{\prime}(x)\right)(k=1, \ldots, n)$, where $b_{j k}^{\prime}=\partial_{j} b_{k}(x)-\partial_{k} b_{j}(x)$;

$\Phi=<\tilde{x} \cdot A \tilde{x}>$;

$\varepsilon(r)$ ro $\varepsilon_{j}(r)(j=1,2, \ldots)$ denotes a positive function which tends to zero as $r \rightarrow \infty$;

$L^{2}(G)$ denotes the class of square integrable functions in the domain $G$ of $\mathbf{R}^{n}$;

$H^{j}(G)(j=1,2)$ denotes the class of $L^{2}$-functions in $G$ such that all distribution derivatives up to $j$ belongs to $L^{2}(G)$;

$L_{l o c}^{2}$ and $H_{l o c}^{j}$ denote the class of locally $L^{2}$ - and $H^{j}$-functions in $\Omega$, respectively;

$C^{j}$ denotes the class of $j$-times continuously differentiable functions;

$Q_{\mu}(\mu>0)$ denotes the class of functions $f(x)$ satisfying the "Stummel condition":

$$
\left\{\begin{array}{l}
\sup _{x \in \Omega} \int_{|x-y|<1}|f(y)|^{2}|x-y|^{-n+4-\mu} d y<\infty \quad \text { if } n \geq 4 \\
\sup _{x \in \Omega} \int_{|x-y|<1}|f(y)|^{2} d y<\infty \quad \text { if } \quad n \leq 3,
\end{array}\right.
$$

where $|x-y|<1$ means the domain $\Omega \cap\{y ;|x-y|<1\}$.

Next we shall state the conditions required on the coefficients of the differential equation (1).

(A1) The $a_{j k}(x)$ are real-valued $C^{2}$-functions in $\Omega$; the $b_{j}(x)$ are real-valued $C^{1}$-functions in $\Omega ; q(x)$ is a real-valued function belonging to $Q_{\mu}$ for some $\mu>0 ; p(x)$ is a complex-valued function belonging to $Q_{\mu}$.

(A2) $a_{j k}(x)=a_{k j}(x)$; there exists a constant $C>1$ such that

$$
C^{-1}|\xi|^{2} \leq \sum_{j, k} a_{j k}(x) \xi_{j} \xi_{k} \leq C|\xi|^{2} \quad \text { for } \quad x \in \Omega, \xi \in \mathbf{R}^{n} .
$$

(A3) (i ) $a_{j k}(x) \rightarrow \delta_{j k}$ as $|x| \rightarrow \infty$;

(ii) $\partial_{l} a_{j k}(x), b_{j k}^{\prime}(x)$ and $p(x)$ behave like $o\left(|x|^{-1}\right)$ as $|x| \rightarrow \infty$; 
(iii) $\partial_{m} \partial_{l} a_{j k}(x)$ behaves like $o\left(|x|^{-1}\right)$ as $|x| \rightarrow \infty$.

(A4) There exist some constants $0<\gamma_{0}<1, \lambda_{0}>0$ and $r_{0}>0$ such that $B\left(r_{0}\right) \subset \Omega$ and for $x \in B\left(r_{0}\right)$

$$
2 \gamma_{0} q(x)+|x| \Phi^{-1}<\tilde{x} \cdot A \operatorname{grad} q(x)>\geq \lambda_{0}
$$

(A5) The unique continuation property holds.

Remark 1.1. If $p(x)$ and $q(x)$ satisfy a Hölder condition except at their singularities and if each connected component of the domain where $p(x)$ and $q(x)$ are regular extends to infinity, then (A5) is satisfied e.g., by a theorem of Landis [7] or Protter [9].

Remark 1.2. If $q(x) \rightarrow \lambda>0$ and $\quad<\tilde{x} \cdot A \operatorname{grad} q(x)>=o\left(|x|^{-1}\right) \quad$ as $|x| \rightarrow \infty$, then (A4) follows. In this case, the following all results can be obtained without (A3, iii).

Now our main purpose is to show the following theorem under the above conditions on the coefficients:

Theorem 1.1. If $u$ is a not identically vanishing solution of (1) in $\Omega$, then we have for any $\gamma>\gamma_{0}$

$$
\liminf _{t \rightarrow \infty} t^{\gamma} \int_{S(t)}\left\{|q(x)||u|^{2}+|<\tilde{x} \cdot A D u>|^{2}\right\} d S=\infty
$$

As a consequence of Theorem 1.1, we can prove the following theorem which is sometimes more convenient for applications:

Theorem 1.2. Let $u$ be a solution of (1) which also satisfies the inequality

$$
\int_{\Omega}(1+|x|)^{\gamma-1}|u(x)|^{2} d x<\infty \quad \text { for some } \gamma>\gamma_{0} .
$$

Then $u$ must identically vanish in $\Omega$.

According to this theorem the uniqueness of $L^{2}$-solutions of (1) follows. Namely, choosing $\gamma=1$ in (1.2), we have the

Corollary 1.1. Let $u$ be a solution of (1) which also belongs to 
$L^{2}(\Omega)$. Then $u=0$ in $\Omega$.

Remark 1.3. In this paper, by a solution $u$ of equation (1) is meant an $H_{l o c}^{2}$, hence $L_{l, c^{-}}^{2}$-function which satisfies (1) in the distribution sense in $\Omega$.

Next we consider the following special equation

$$
-\Delta u-q(x) u=0 \quad \text { in } \Omega,
$$

where $\Delta$ is the Laplacian and $q(x) \in Q_{\mu}$ and satisfies the following condition:

(A4)' There exist constants $0<\gamma_{0} \leq 1, \lambda_{0}>0$ and $r_{0}>0$ such that $B\left(r_{0}\right) \subset \Omega$ and for $x \in B\left(r_{0}\right)$

$$
2 \gamma_{0} q(x)+|x|<\tilde{x} \cdot \operatorname{grad} q(x)>\geq \lambda_{0} .
$$

In this case we can change the result of Theorem 1.1 as follows:

Theorem 1.3. If $u$ is a not identically vanishing solution of (1.3) in $\Omega$, then we have

$$
\liminf _{t \rightarrow \infty} t^{\gamma o} \int_{S(t)}\left\{(1+|q(x)|)|u|^{2}+|<\tilde{x} \cdot \operatorname{grad} u>|^{2}\right\} d S>0
$$

Remark 1.4. Making use of this theorem, we can also obtain a result corresponding to Theorem 1.2: Let $u$ be a solution of (1.3) which also satisfies the inequality

$$
\int_{\Omega}(1+|x|)^{\gamma_{0}-1}|u(x)|^{2} d x<\infty .
$$

Then $u=0$ in $\Omega$ (cf. Uchiyama [14], Theorem 2.1).

We shall prove Theorem 1.1 in $\S 3$, Theorem 1.2 in $\$ 4$ and Theorem 1.3 in $\S 5$. $\S 2$ will be devoted to obtain apriori estimates related to equation (1).

\section{§2. Preliminaries}

Let $u$ be a solution of (1): 


$$
-<D \cdot A D u>-q u+p u=0
$$

Let $\rho(r)$ be a real-valued, non-decreasing $C^{3}$-function of $r>r_{0}$, and put

$$
v(x)=e^{\rho(r)} u(x) \quad(r=|x|) .
$$

Then it readily follows from (2.1) that $v$ satisfies in $B\left(r_{0}\right)$ the equation

$$
-<D \cdot A D v>+2 \rho^{\prime}<\tilde{x} \cdot A D v>-\tilde{q} v+\tilde{p} v=0
$$

$$
\begin{aligned}
& \tilde{q}=q+\Phi\left(\rho^{\prime 2}-\rho^{\prime \prime}-\frac{n-1}{r} \rho^{\prime}\right), \\
& \tilde{p}=p+\left(\operatorname{div}(A \tilde{x})-\frac{n-1}{r} \Phi\right) \rho^{\prime},
\end{aligned}
$$

where $\rho^{\prime}=d \rho / d r$ and $\rho^{\prime \prime}=d^{2} \rho / d r^{2}$.

We shall first prove two equalities which are satisfied for solutions $v$ of equation (2.3).

Proposition 2.1. Let $\psi(x)$ be a real-valued $C^{1}$-function of $x \in B\left(r_{0}\right)$. Then we have for $t>s>r_{0}$

$$
\begin{aligned}
& \int_{B(s, t)} \psi\left(<A D v \cdot \widetilde{D v}>-\tilde{q}|v|^{2}\right) d x \\
& =\left[\int_{S(t)}-\int_{S(s)}\right] \psi \operatorname{Re}[<\tilde{x} \cdot A D v>\bar{v}] d S \\
& \quad-\int_{B(s, t)} \operatorname{Re}\left[<\left(\operatorname{grad} \psi+2 \rho^{\prime} \psi \tilde{x}\right) \cdot A D v>\bar{v}-\psi \tilde{p}|v|^{2}\right] d x,
\end{aligned}
$$

where $\operatorname{Re}[f]$ means the real part of $f$.

Proof. (2.6) can be obtained by the integration by parts of (2.3) multiplied by $\psi \bar{v}$.

q.e.d.

Proposition 2.2. Let $\gamma$ be a real number. Then we have for $t>s>r_{0}$ 
(2.7)

$$
\begin{aligned}
& {\left[\int_{S(t)}-\int_{S(s)}\right] r^{\gamma}\left\{\Phi^{-1}|<\tilde{x} \cdot A D v>|^{2}-\frac{1}{2}\left(<A D v \cdot \overline{D v}>-\tilde{q}|v|^{2}\right)\right\} d s} \\
& =\int_{B(s, t)} r^{\gamma-1}\left\{\left(1-\gamma_{0}\right)<A D v \cdot \overline{D v}>-(1-\gamma) \Phi^{-1}|<\tilde{x} \cdot A D v>|^{2}\right\} d x \\
& \quad+\frac{1}{2} \int_{B(s, t)} r^{\gamma-1}\left(2 \gamma_{0} \tilde{q}+r \Phi^{-1}<\tilde{x} \cdot A \operatorname{grad} \tilde{q}>\right)|v|^{2} d x \\
& \quad-\frac{1}{2} \int_{B(s, t)} \Psi\left(<A D v \cdot \overline{D v}>-\tilde{q}|v|^{2}\right) d x \\
& \quad+\int_{B(s, t)} 2 \rho^{\prime} r^{\gamma} \Phi^{-1}|<\tilde{x} \cdot A D v>|^{2} d x \\
& \quad+\int_{B(s, t)} r^{\gamma} \Phi^{-1}\left(K_{1}+K_{2}+K_{3}+K_{4}\right) d x
\end{aligned}
$$

where

$$
\begin{gathered}
\Psi=r^{\gamma-1}\left\{\gamma-2 \gamma_{0}+r \Phi^{-1} \operatorname{div}(A \tilde{x})+r<\tilde{x} \cdot A \operatorname{grad} \Phi^{-1}>\right\} \\
K_{1}=\operatorname{Re} \sum_{l}[A D v]_{l}\left(<\tilde{x} \cdot A_{l}^{\prime} \overline{D v}>-i<\tilde{x} \cdot A B_{l}^{\prime} \bar{v}>\right) \\
-\frac{1}{2} \operatorname{Re} \sum_{l}[A \tilde{x}]_{l}<A_{l}^{\prime} \overline{D v} \cdot D v>
\end{gathered}
$$

(2.10) $\quad K_{2}=r^{-1}\left(|A D v|^{2}-\Phi<A D v \cdot \overline{D v}>\right)$,

(2.11) $K_{3}=\operatorname{Re}\left[\Phi<\operatorname{grad} \Phi^{-1} \cdot A D v><\tilde{x} \cdot A \overline{D v}>\right]$,

(2.12) $\quad K_{4}=\operatorname{Re}[\tilde{p} v<\tilde{x} \cdot A \overline{D v}>]$.

Proof. We multiply the both sides of (2.3) by $\langle\tilde{x} \cdot A D v\rangle$ and take the real parts. Then we have

(2.13) $-\operatorname{Re}[(<D \cdot A D v>+\tilde{q} v)<\tilde{x} \cdot A \overline{D v}>]+2 \rho^{\prime}|<\tilde{x} \cdot A D v>|^{2}+K_{4}=0$.

The first term of the left side becomes

$$
\begin{aligned}
& -\operatorname{Re}[(<D \cdot A D v>+\tilde{q} v)<\tilde{x} \cdot A \overline{D v}>] \\
= & -\operatorname{Re} \operatorname{div}\left[A D v<\tilde{x} \cdot A \overline{D v}>-\frac{1}{2} A \tilde{x}\left(<A D v \cdot \overline{D v}>-\tilde{q}|v|^{2}\right)\right]
\end{aligned}
$$




$$
\begin{aligned}
& +\left(1-\gamma_{0}\right) r^{-1} \Phi<A D v \cdot \overline{D v}>-r^{-1}|<\tilde{x} \cdot A D v>|^{2}+K_{2} \\
& +\frac{1}{2}\left(2 \gamma_{0} r^{-1} \Phi \tilde{q}+<\tilde{x} \cdot A \operatorname{grad} \tilde{q}>\right)|v|^{2} \\
& -\frac{1}{2}\left\{\operatorname{div}(A \tilde{x})-2 \gamma_{0} r^{-1} \Phi\right\}\left(<A D v \cdot \overline{D v}>-\tilde{q}|v|^{2}\right)+K_{1} .
\end{aligned}
$$

Thus, multiplying (2.13) by $r^{\gamma} \Phi^{-1}$ and integrating on $B(s, t)$, we have (2.7).

q.e.d.

Next we shall estimate the right side of (2.7). By (A3, i, ii) it follows that there exists a function $\varepsilon(r)$ verifying

$$
\left|\partial_{j} \Phi\right|+\left|\partial_{j} \Phi^{-1}\right|+\left|\operatorname{div}(A \tilde{x})-\frac{n-1}{r} \Phi\right| \leq \varepsilon(r) r^{-1} .
$$

Further it follows from $(\mathrm{A} 3, \mathrm{i})$ that

$$
\|\left. A D v\right|^{2}-\Phi<A D v \cdot \overline{D v}>\mid \leq \varepsilon(r) \Phi<A D v \cdot \overline{D v}>
$$

In view of these inequalities, using again (A3, i, ii) and noting the inequality

$$
\Phi^{-1}|<\tilde{x} \cdot A D v>|^{2} \leq<A D v \cdot \overline{D v}>
$$

we obtain the following

Lemma 2.1. There exists a function $\varepsilon_{1}(r)$ such that

$$
\left|K_{1}\right|+\left|K_{2}\right|+\left|K_{3}\right| \leq \varepsilon_{1}(r) r^{-1} \Phi\left(<A D v \cdot \overline{D v}>+|v|^{2}\right) \text {. }
$$

Since the inequality

$$
|\tilde{p}| \leq \varepsilon(r) r^{-1}\left(1+\rho^{\prime}\right)
$$

follows from (2.5), (A3, ii) and (2.14), we have similarly the

Lemma 2.2. There exists a function $\varepsilon_{2}(r)$ such that

$$
\begin{aligned}
\left|K_{4}\right| \leq & \varepsilon_{2}(r) r^{-1}\left(|<\tilde{x} \cdot A D v>|^{2}+\Phi|v|^{2}\right) \\
& +\varepsilon_{2}(r) \rho^{\prime}\left(|<\tilde{x} \cdot A D v>|^{2}+r^{-2} \Phi|v|^{2}\right) .
\end{aligned}
$$


Lemma 2.3. There exist a constant $C_{1}>0$ and $a$ function $\varepsilon_{3}(r)$ such that

$$
|\Psi| \leq C_{1} r^{\gamma-1}
$$$$
|\operatorname{grad} \Psi| \leq \varepsilon_{3}(r) r^{\gamma-1}
$$

Proof. (2.20) follows (2.8) and (2.14). (2.21) follows if we note (A3, iii) also.

q.e.d.

Applying the inequalities of this lemma to equality (2.6) of proposition 2.1 with $\psi=\Psi$, we obtain the following

Lemma 2.4. There exist functions $\varepsilon_{4}(r)$ and $\varepsilon_{5}(r)$ such that

$$
\begin{aligned}
& \int_{B(s, t)} \Psi\left(<A D v \cdot \overline{D v}>-\tilde{q}|v|^{2}\right) d x \\
& \leq\left[\int_{S(t)}-\int_{S(s)}\right] \Psi \operatorname{Re}[<\tilde{x} \cdot A D v>\bar{v}] d S \\
& \quad+\int_{B(s, t)} \varepsilon_{4}(r) r^{\cdot \gamma-1}\left(<A D v \cdot \overline{D v}>+|v|^{2}\right) d x \\
& \quad+\int_{B(s, t)} \rho^{\prime} r^{\gamma}\left\{\Phi^{-1}|<\tilde{x} \cdot A D v>|^{2}+\left(C_{1}^{2}+\varepsilon_{5}(r)\right) r^{-2}|v|^{2}\right\} d x .
\end{aligned}
$$

Lemma 2.5. Let $\alpha$ and $\beta$ be non-negative constants. Then there exists a function $\varepsilon_{6}(r)$ such that

$$
\begin{aligned}
& {\left[\int_{S(t)}-\int_{S(s)}\right]\left(\alpha r^{\gamma-1}-\beta r^{\gamma-2}\right) \Phi|v|^{2} d S} \\
& \geq-\int_{B(s, t)}\left\{\alpha+(\alpha+\beta) \varepsilon_{6}(r)\right\} r^{\gamma-1}\left(\Phi^{-1}|<\tilde{x} \cdot A D v>|^{2}+|v|^{2}\right) d x .
\end{aligned}
$$

Proof. We have for any $v \in H_{l o c}^{1}$

$$
\begin{aligned}
\operatorname{div} & {\left[\left(\alpha r^{\gamma-1}-\beta r^{\gamma-2}\right) A \tilde{x}|v|^{2}\right]=\operatorname{Re}\left[2\left(\alpha r^{\gamma-1}-\beta r^{\gamma-2}\right)<\tilde{x} \cdot A D v>\bar{v}\right] } \\
& +\left\{\frac{\alpha(\gamma-1)}{r}-\frac{\beta(\gamma-2)}{r^{2}}+\left(\alpha-\frac{\beta}{r}\right) \Phi^{-1} \operatorname{div}(A \tilde{x})\right\} r^{\gamma-1} \Phi|a|^{2} .
\end{aligned}
$$


Thus the integration on $B(s, t)$ and the Schwarz inequality yield (2.23).

q.e.d.

Now, summarizing the above results, we can prove the following proposition:

Proposition 2.3. Let $\gamma$ be a constant such that $\gamma_{0}<\gamma \leq 1$. Then we have for $t>s>r_{0}$

$$
\begin{aligned}
& {\left[\int_{S(t)}-\int_{S(s)}\right] r \cdot\left\{\Phi^{-1}|<\tilde{x} \cdot A D v>|^{2}-\frac{1}{2}\left(<A D v \cdot \overline{D v}>-\tilde{q}|v|^{2}\right)\right\} d s} \\
& \quad+\frac{1}{2}\left[\int_{S(t)}-\int_{S(s)}\right]\left\{\Psi \operatorname{Re}[<\tilde{x} \cdot A D v>\bar{v}]+\left(\alpha r^{\gamma-1}-\beta r^{\gamma-2}\right) \Phi|v|^{2}\right\} d s \\
& \geq \int_{B(s, t)}\left(\gamma-\gamma_{0}-\varepsilon_{7}(r)\right) r^{\gamma-1}<A D v \cdot \overline{D v}>d x \\
& \quad+\int_{B(s, t)}\left\{\left(\frac{3}{2}-\varepsilon_{2}(r)\right) \rho^{\prime}-\frac{\alpha}{2 r}\right\} r^{\gamma} \Phi^{-1}|<\tilde{x} \cdot A D v>|^{2} d x \\
& \quad+\frac{1}{2} \int_{B(s, t)} r^{\gamma-1}\left(2 \gamma_{0} \tilde{q}+r \Phi^{-1}<\tilde{x} \cdot A \operatorname{grad} \tilde{q}>\right)|v|^{2} d x \\
& \quad-\int_{B(s, t)} r^{\gamma-1}\left\{\left(\varepsilon_{7}(r)+\frac{\alpha}{2}\right)+\left(\frac{1}{2} C_{1}^{2}+\varepsilon_{8}(r)\right) \rho^{\prime} r^{-1}\right\}|v|^{2} d x
\end{aligned}
$$

where $\varepsilon_{7}=\varepsilon_{1}+\varepsilon_{2}+\frac{1}{2} \varepsilon_{4}+\frac{1}{2}(\alpha+\beta) \varepsilon_{6}$ and $\varepsilon_{8}=\varepsilon_{2}+\frac{1}{2} \varepsilon_{5}$.

Proof. It follows from the condition on $\gamma$ and (2.16) that the first term of the right of equality (2.7) is estimated from below by the integral

$$
\int_{B(s, t)}\left(\gamma-\gamma_{0}\right) r^{\gamma-1}<A D v \cdot \overline{D v}>d x
$$

Then, applying Lemmas $2.1,2,4$ and 5 to (2.7), we have (2.24). q.e.d.

Next, we shall derive another inequality which follows from the 
ellipticity of the differential operator $\langle D \cdot A D\rangle$ and the condition that $\tilde{p}, \tilde{q} \in Q_{\mu}(\mu>0)$.

Proposition 2.4. Suppose that $\rho^{\prime}$ and $\rho^{\prime \prime}$ in equation (2.3) are bounded in $r>r_{0}$. Let $v$ be a solution of (2.3) which also belongs to $L^{2}\left(B\left(r_{0}\right)\right)$. Then we have

$$
\int_{B\left(r_{0}+1\right)}\left\{<A D v \cdot \overline{D v}>+(|\tilde{q}|+|\tilde{p}|)|v|^{2}\right\} d x \leq C_{2} \int_{B\left(r_{0}\right)}|v|^{2} d x
$$

for some $C_{2}>0$.

Proof. The assertion for equation (2.3) with $\rho^{\prime}=0$ has been essentially proved in Ikebe-Kato [5]. The existence of the term $2 \rho^{\prime}<\tilde{x}$. $A D v>$ causes no serious difficulty since we have for any $\varepsilon>0$

$$
\begin{aligned}
\int_{B\left(r_{0}+1\right)} 2 \rho^{\prime}|<\tilde{x} \cdot A D v>\bar{v}| d x \\
\leq \varepsilon \int_{B\left(r_{0}+1\right)}<A D v \cdot \overline{D v}>d x+C(\varepsilon) \int_{B\left(r_{0}+1\right)}|v|^{2} d x,
\end{aligned}
$$

where $C(\varepsilon)$ is a positive constant depending on $\varepsilon$ (cf., [5]; Lemmas 2 and 5).

q.e.d.

\section{§3. Proof of Theorem 1.1}

In this section the proof of Theorem 1.1 will be given by means of a series of lemmas (cf., Roze [10] or Éidus [3]; § 2).

Lemma 3.1. Let $"$ be a solution of (1) satisfying also the condition

$$
\liminf _{t \rightarrow \infty} t^{\gamma} \int_{S(t)}\left(|q \| u|^{2}+|<\tilde{x} \cdot A D u>|^{2}\right) d S=0
$$

for some $\gamma>\gamma_{0}$. Then we have for any $m>0$

$$
\int_{B\left(r_{0}\right)} r^{\prime \prime \prime}\left(|u|^{2}+|D u|^{2}\right) d x<\infty
$$


Proof. Without loss of generality we can assume that $\gamma_{0}<\gamma \leq 1$. We put $\rho(r)=0, \alpha=0$ and $\beta=1$ in inequality (2.24). Then $v=u$ and $\tilde{q}=q$. Since

$$
\begin{gathered}
\int_{B(s, t)} r^{\gamma-1}\left(2 \gamma_{0} q+r \Phi^{-1}<\tilde{x} \cdot A \operatorname{grad} q>\right)|u|^{2} d x \\
\geq \lambda_{0} \int_{B(s, t)} r^{\gamma-1}|u|^{2} d x
\end{gathered}
$$

by (A4), it follows from (2.16), (2.20) and (2.24) that

$$
\begin{aligned}
\frac{1}{2} \int_{S(t)} r^{\gamma}\left(\Phi^{-1} \mid\right. & <\tilde{x} \cdot A D u>\left.\right|^{2}+|q||u|^{2} \\
& \left.+C_{1} r^{-1}|<\tilde{x} \cdot A D u>\bar{u}|-r^{-2}|u|^{2}\right) d S \\
+\frac{1}{2} \int_{S(s)} r^{\gamma}( & <A D u \cdot \overline{D u}>-q|u|^{2} \\
& \left.+C_{1} r^{-1}|<\tilde{x} \cdot A D u>\bar{u}|+r^{-2}|u|^{2}\right) d S \\
\geq \int_{B(s, t)} r^{\gamma-1}(\gamma & \left.-\gamma_{0}-\varepsilon_{7}(r)\right)<A D u \cdot \overline{D u}>d x \\
& +\frac{1}{2} \int_{B(s, t)} r^{\gamma-1}\left(\lambda_{0}-2 \varepsilon_{7}(r)\right)|u|^{2} d x .
\end{aligned}
$$

By the Schwarz inequality

$$
C_{1} r^{-1}|<\tilde{x} \cdot A D u>\bar{u}|-r^{-2}|u|^{2} \leq \frac{1}{4} C_{1}^{2}|<\tilde{x} \cdot A D u>|^{2} .
$$

We choose $r_{1} \geq r_{0}$ large so that for $r>r_{1}$

$$
\gamma-\gamma_{0}-\varepsilon_{7}(r) \geq\left(\gamma-\gamma_{0}\right) / 2, \quad \lambda_{0}-2 \varepsilon_{7}(r) \geq \lambda_{0} / 2 .
$$

Then, letting $t \rightarrow \infty$ in (3.3), we see by (3.1) and (3.4) that for $s>r_{1}$

$$
\begin{aligned}
& \int_{S(s)} r^{\gamma}\left(<A D u \cdot \overline{D u}>-q|u|^{2}\right) d S \\
& \quad+\int_{S(s)}\left(C_{1} r^{\gamma-1}|<\tilde{x} \cdot A D u>\bar{u}|+r^{\nu-2}|u|^{2}\right) d S \\
& \geq \int_{R(s)} r r^{\gamma-1}\left\{\left(\gamma-\gamma_{0}\right)<A D u \cdot \overline{D u}>+\frac{1}{2} \lambda_{0}|u|^{2}\right\} d x .
\end{aligned}
$$


Integrating this inequality with respect to $s$ from $t$ to $t_{1}$, where $r_{1}$ $<t<t_{1}$, and using equality (2.6) with $\psi=r^{\gamma}$, we obtain

$$
\begin{aligned}
& \int_{t}^{t_{1}} d s \int_{B(s)} r^{\gamma-1}\left\{\left(\gamma-\gamma_{0}\right)<A D u \cdot \overline{D u}>+\frac{1}{2} \lambda_{0}|u|^{2}\right\} d x \\
& \leq\left[\int_{S\left(t_{1}\right)}+\int_{S(t)}\right] r^{\gamma}|<\tilde{x} \cdot A D u>\bar{u}| d S \\
& \quad+\int_{B\left(t, t_{1}\right)} r^{\gamma-1}\left\{\left(C_{1}+\gamma\right)|<\tilde{x} \cdot A D u>\bar{u}|+\left(r^{-1}+r|p|\right)|u|^{2}\right\} d x .
\end{aligned}
$$

Inequality (3.5) implies that

$$
\liminf _{t_{1} \rightarrow \infty} t_{1}^{\gamma} \int_{S\left(t_{1}\right)}|<\tilde{x} \cdot A D u>\bar{u}| d S=0 .
$$

Hence

$$
\begin{aligned}
& \int_{B(t)}(r-t) r^{\gamma-1}\left\{\left(\gamma-\gamma_{0}\right)<A D u \cdot \overline{D u}>+\frac{\lambda_{0}}{2}|u|^{2}\right\} d x \\
& \leq \int_{S(t)} r^{\gamma}\left(|<\tilde{x} \cdot A D u>|^{2}+|u|^{2}\right) d S \\
& \quad+\left(C_{1}+\gamma+\varepsilon(t)\right) \int_{B(t)} r^{\gamma-1}\left(|<\tilde{x} \cdot A D u>|^{2}+|u|^{2}\right) d x<\infty .
\end{aligned}
$$

Repeating the integration with respect to $t$, we see that the assertion of the lemma is valid for arbitrary $m>0$.

q.e.d.

Lemma 3.2. Let $u$ be a solution of (1) satisfying also condition (3.1) for some $\gamma>\gamma_{0}$. Then we have for any $k>0$

$$
\int_{B\left(r_{0}\right)} e^{k r^{1-v}}|u|^{2} d x<\infty
$$

where $v$ is a constant such that $0<v<1$.

Proof. Let $\rho(r)=m \log r(m \geq n), 0<\alpha \leq \min \left\{\lambda_{0} / 3,1\right\}$ and $\beta=0$ in inequality (2.24). Then $v=r^{m m} u, \rho^{\prime}=\frac{m}{r}$ and $\tilde{q}=q+\Phi \frac{m(m-n+2)}{r^{2}}$. It follows that there exists a function $\varepsilon_{9}(r)$ verifying 


$$
2 \gamma_{0} \tilde{q}+r \Phi^{-1}<\tilde{x} \cdot A \operatorname{grad} \tilde{q}>\geq \lambda_{0}-2\left(1-\gamma_{0}+\varepsilon_{9}(r)\right) \frac{m(m-n+2)}{r^{2}}
$$

If we choose $r_{2} \geq r_{0}$ sufficiently large, then for $r>r_{2}$

$$
\left\{\begin{array}{l}
\gamma-\gamma_{0}-\varepsilon_{7}(r) \geq 0 \\
\left(\frac{3}{2}-\varepsilon_{2}(r)\right) \frac{m}{r}-\frac{\alpha}{2 r} \geq 0 \\
\lambda_{0}-2 \varepsilon_{7}(r)-\alpha \geq \lambda_{0} / 2 \\
1-\gamma_{0}+\varepsilon_{9}(r) \leq 2\left(1-\gamma_{0}\right) \\
\frac{1}{2} C_{1}^{2}+\varepsilon_{8}(r) \leq C_{1}^{2}
\end{array}\right.
$$

Further, since $r^{\gamma / 2} v \in L^{2}\left(B\left(r_{0}\right)\right)$ by (3.2), it follows from (2.25) that

$$
\liminf _{t \rightarrow \infty} t^{\gamma} \int_{S(t)}\left\{(1+|\tilde{q}|)|v|^{2}+|<\tilde{x} \cdot A D v>|^{2}\right\} d S=0
$$

Thus, in view of the above inequalities, letting $t \rightarrow \infty$ in (2.24), we have

$$
\begin{gathered}
\text { (3.8) }-\int_{S(s)} r^{\gamma}\left(2 \Phi^{-1}|<\tilde{x} \cdot A D v>|^{2}-\frac{C_{1}}{r}|<\tilde{x} \cdot A D v>\bar{v}|+\frac{\alpha}{r} \Phi|v|^{2}\right) d S \\
+\int_{S(s)} r^{\gamma}\left(<A D v \cdot \overline{D v}>-\tilde{q}|v|^{2}\right) d S \\
\geq \int_{B(s)} r^{\gamma-1}\left\{\frac{\lambda_{0}}{2}-4\left(1-\gamma_{0}\right) \frac{m(m-n+2)}{r^{2}}-2 C_{1}^{2} \frac{m}{r^{2}}\right\}|x|^{2} d x
\end{gathered}
$$

for $s>r_{2}$. Multiply the both sides of (3.8) by $s^{-2 m}$ and integrate with respect to $s$ from $t$ to $\infty$, where $t>r_{2}$. Then we obtain

$$
\text { (3.9) } \begin{gathered}
-\int_{B(t)} r^{\gamma-2 m}\left\{\Phi^{-1}|<\tilde{x} \cdot A D v>|^{2}+\left(\frac{\alpha}{r}-\left(\frac{C_{1}}{2 r}\right)^{2}\right) \Phi|v|^{2}\right\} d x \\
+\int_{B(t)} r^{\gamma-2 m}\left(<A D v \cdot \overline{D v}>-\tilde{q}|v|^{2}\right) d x \\
\geq \int_{t}^{\infty}\left\{\frac{\lambda_{0}}{2}-4\left(1-\gamma_{0}\right) \frac{m(m-n+2)}{s^{2}}-2 C_{1}^{2} \frac{m}{s^{2}}\right\} s^{-2 m} d s \int_{B(s)} r^{\gamma-1}|v|^{2} d x .
\end{gathered}
$$


By (2.6)

$$
\begin{aligned}
& \int_{B(t)} r^{\gamma-2 m}\left(<A D v \cdot \overline{D v}>-\tilde{q}|v|^{2}\right) d x \\
& =-\int_{S(t)} r^{\gamma-2 m} \operatorname{Re}[<\tilde{x} \cdot A D v>\bar{v}] d S \\
& \quad-\int_{B(t)} r^{\gamma-2 m-1}\left(\gamma \operatorname{Re}[<\tilde{x} \cdot A D v>\bar{v}]-r \operatorname{Re}[\tilde{p}]|v|^{2}\right) d x,
\end{aligned}
$$

where

$$
\begin{aligned}
& -\int_{S(t)} r^{\gamma-2 m} \operatorname{Re}[<\tilde{x} \cdot A D v>\bar{v}] d S \\
& =-\frac{1}{2} \frac{d}{d t} \int_{S(t)} r^{\gamma-2 m} \Phi|v|^{2} d S \\
& +\frac{1}{2} \int_{S(t)}\left(\gamma-2 m+r \Phi^{-1} \operatorname{div}(A \tilde{x})\right) r^{\gamma-2 m-1} \Phi|v|^{2} d S .
\end{aligned}
$$

Hence, by (3.10),

$$
\begin{aligned}
& \int_{B(t)} r^{\gamma-2 m}\left(<A D v \cdot \overline{D v}>-\tilde{q}|v|^{2}\right) d x \\
& \leq-\frac{1}{2} \frac{d}{d t} \int_{S(t)} r^{\gamma-2 m} \Phi|v|^{2} d S \\
& \quad-\frac{1}{2} \int_{S(t)}\left(2 m-\gamma-n+1-\varepsilon_{10}(r)\right) r^{\gamma-2 m-1} \Phi|v|^{2} d S \\
& \quad+\frac{1}{2} \int_{B(t)} r^{\gamma-2 m}\left\{\Phi^{-1}<|\tilde{x} \cdot A D v|^{2}+\left(\left(\frac{\gamma}{r}\right)^{2}+\frac{1}{r} \varepsilon_{11}(r)\right) \Phi|v|^{2}\right\} d x .
\end{aligned}
$$

Substituting this in (3.9), we obtain

$$
\begin{aligned}
& -\frac{1}{2} \int_{B(t)} r^{\gamma-2 m}\left\{\Phi^{-1}|<\tilde{x} \cdot A D v>|^{2}\right. \\
& \left.+\left(\frac{2 \alpha}{r}-\left(\frac{C_{1}}{r}\right)^{2}-\left(\frac{\gamma}{r}\right)^{2}-\frac{1}{r} \varepsilon_{11}(r)\right) \Phi|v|^{2}\right\} d x \\
& -\frac{1}{2}\left[\frac{d}{d t} \int_{S(t)} r^{\gamma-2 m} \Phi|v|^{2} d S+\frac{m}{t} \int_{S(t)} r^{-2 m} \Phi|v|^{2} d s\right]-
\end{aligned}
$$




$$
\begin{aligned}
& -\frac{1}{2} \int_{S(t)}\left(m-\gamma-n+1-\varepsilon_{10}(r)\right) r^{\gamma-2 m-1} \Phi|v|^{2} d S \\
& \geq \int_{1}^{\infty}\left\{\frac{\lambda_{0}}{2}-4\left(1-\gamma_{0}\right) \frac{m(m-n+2)}{s^{2}}-2 C_{1}^{2} \frac{m}{s^{2}}\right\} s^{-2 m} d S \times \\
& \times \int_{B(s)} r^{\gamma-1}|v|^{2} d x .
\end{aligned}
$$

We fix arbitrary $k>0$ and $0<v<1$, and set $m=k(1-v) t^{1-v}$. Then for $r \geq t>r_{3}$, where $r_{3} \geq r_{2}$ is sufficiently large, we have

$$
\left\{\begin{array}{l}
\frac{\alpha}{r}-\left(\frac{C_{1}}{r}\right)^{2}-\left(\frac{\gamma}{r}\right)^{2}-\frac{1}{r} \varepsilon_{11}(r) \geq 0 \\
m-\gamma-n+1-\varepsilon_{10}(r) \geq 0 \\
\frac{\lambda_{0}}{2}-4\left(1-\gamma_{0}\right) \frac{m(m-n+2)}{r^{2}}-2 C_{1}^{2} \frac{m}{r^{2}} \geq 0
\end{array}\right.
$$

Hence, putting

$$
F(t)=\int_{S(t)} r^{\gamma-2 m} \Phi|v|^{2} d S=\int_{S_{(t)}} r^{\gamma} \Phi|u|^{2} d S
$$

we have from (3.11)

$$
\frac{d}{d t} F(t)+k(1-v) t^{-v} F(t) \leq 0 \text { for } t>r_{3}
$$

Therefore, for any $k>0$ and $0<v<1$

$$
F(t) \leq C e^{-k t^{1-v}}
$$

where $C>0$ is independent of $t$. This implies (3.6) and the proof is completed.

q.e.d.

Proof of Theorem 1.1. We shall prove the following assertion which is equivalent to Theorem 1.1; Let $u$ be $a$ solution of (1) satisfying also condition (3.1) for some $\gamma>\gamma_{0}$. Then $u$ must identically vanish in $\Omega$.

We return once more to inequality (2.24). We put $\rho(r)=k r^{1-v}$ 
and $\alpha=\beta=0$. Then $v=e^{k r^{1-v}} u$ and $\rho^{\prime}=k(1-v) / r^{v}$. Since

$$
\tilde{q}=q+\Phi\left\{\frac{k^{2}(1-v)^{2}}{r^{2 v}}-\frac{k(1-v)(n-1-v)}{r^{1+v}}\right\},
$$

it follows from (A4) that

$$
\begin{aligned}
2 \gamma_{0} \tilde{q}+r \Phi^{-1}<\tilde{x} \cdot A \operatorname{grad} \tilde{q}> & \\
\geq & \lambda_{0}+2\left(\gamma_{0}-v-\varepsilon(r)\right) \frac{k^{2}(1-v)^{2}}{r^{2 v}} \\
& +\left(1+v-2 \gamma_{0}-\varepsilon(r)\right) \frac{k(1-v)(n-1-v)}{r^{1+v}}
\end{aligned}
$$

If we choose $r_{4} \geq r_{0}$ sufficiently large, then for $r>r_{4}$

$$
\left\{\begin{array}{l}
\gamma-\gamma_{0}-\varepsilon_{7}(r) \geq 0 \\
\frac{3}{2}-\varepsilon_{2}(r) \geq 0 \\
\lambda_{0}-2 \varepsilon_{7}(r) \geq \frac{\lambda_{0}}{2} \\
\frac{1}{2} C_{1}^{2}+\varepsilon_{8}(r) \leq C_{1}^{2}
\end{array}\right.
$$

By Lemma $3.2 r^{\gamma / 2} v \in L^{2}\left(B\left(r_{0}\right)\right)$. Thus, by means of inequality (2.25), we see

$$
\liminf _{t \rightarrow \infty} t^{\gamma} \int_{S(t)}\left\{(1+|\tilde{q}|)|v|^{2}+|D v|^{2}\right\} d S=0
$$

Using the above inequalities and letting $t \rightarrow \infty$ in (2.24), we then have for $s>r_{4}$

$$
\begin{aligned}
& \text { (3.14) }-\frac{s^{\gamma}}{2} \int_{S(s)}\left\{2 \Phi^{-1}|<\tilde{x} \cdot A D v>|^{2}-<A D v \cdot \overline{D v}\right\rangle \\
& \left.+\tilde{q}|v|^{2}-|\Psi<\tilde{x} \cdot A D v>\bar{v}|\right\} d S \\
& \geq \frac{1}{2} \int_{B(s)} r^{\gamma-1}\left\{\frac{\lambda_{0}}{2}+2\left(\gamma_{0}-v-\varepsilon(r)\right) \frac{k^{2}(1-v)^{2}}{r^{2 v}}+\right.
\end{aligned}
$$




$$
\left.+\left(1+v-2 \gamma_{0}-\varepsilon(r)\right) \frac{k(1-v)(n-1-v)}{r^{1+v}}-C_{1}^{2} \frac{k(1-v)}{r^{1+v}}\right\}|v|^{2} d x
$$

Now we choose $v$ less than $\gamma_{0}: 0<v<\gamma_{0}$. Then, obviously, there exists an $r_{5} \geq r_{4}$ such that for any $k \geq 1$ and $s>r_{5}$

$$
\begin{aligned}
\int_{S(s)}\left\{2 \Phi^{-1}|<\tilde{x} \cdot A D v>|^{2}-<A D v \cdot \overline{D v}>\right. \\
\left.+\tilde{q}|v|^{2}-|\Psi<\tilde{x} \cdot A D v>\bar{v}|\right\} d S \leq 0 .
\end{aligned}
$$

Since

$$
\begin{aligned}
|<\tilde{x} \cdot A D v>|^{2}=e^{2 k r^{1-v}}\left\{|<\tilde{x} \cdot A D u>|^{2}\right. \\
\left.\quad+\frac{2 k(1-v)}{r^{v}} \Phi \operatorname{Re}[<\tilde{x} \cdot A D u>\bar{u}]+\frac{k^{2}(1-v)^{2}}{r^{2 v}} \Phi^{2}|u|^{2}\right\}
\end{aligned}
$$

and

$$
\begin{aligned}
& <A D v \cdot \overline{D v}>=e^{2 k r^{1-v}}\{<A D u \cdot \overline{D u}> \\
& \left.\quad+\frac{2 k(1-v)}{r^{v}} \operatorname{Re}[<\tilde{x} \cdot A D u>\bar{u}]+\frac{k^{2}(1-v)^{2}}{r^{2 v}} \Phi|u|^{2}\right\},
\end{aligned}
$$

We can write the left side of (3.15) in the form

$$
e^{2 k s^{1-v}}\left\{k^{2} M_{1}(s)+k M_{2}(s)+M_{3}(s)\right\},
$$

where

$$
M_{1}(s)=\frac{(1-v)^{2}}{s^{2 v}} \int_{S(s)} \Phi|u|^{2} d S
$$

and $M_{2}(s)$ and $M_{3}(s)$ are independent of $k$. Suppose that $M_{1}(s)>0$ for some $s>r_{5}$. Then $k$ can be chosen so large that (3.15) is nolonger valid. Hence $u=0$ in $B\left(r_{5}\right)$. By the unique continuation property (A5), it follows that $u=0$ in $\Omega$. This concludes the proof.

q.e.d. 


\section{§4. Proof of Theorem 1.2}

Suppose that a solution $u$ of (1) satisfies condition (1.2) of Theorem 1.2. Then, by (2.25) of Proposition 2.4, it follows that

$$
\liminf _{t \rightarrow \infty} t^{\gamma} \int_{S(t)}\left\{(1+|q|)|u|^{2}+|D u|^{2}\right\} d S=0
$$

This contradicts (1.1) of Theorem 1.1 and hence we see that $u=0$ in $\Omega$. This concludes the proof.

\section{$\S 5$. Proof of Theorem 1.3}

Let $u$ be a solution of the simpler equation (1.3). We put $\gamma=\gamma_{0}$ in Proposition 2.2. Then, noting that $K_{1}=K_{2}=K_{3}=K_{4}=0$ and $\Psi$ $=\left(n-1-\gamma_{0}\right) r^{\gamma_{0}-1}$, and applying Proposition 2.1 with $\psi=\left(n-1-\gamma_{0}\right) r^{\gamma_{0}-1}$, we have

$$
\begin{aligned}
& {\left[\int_{S(t)}-\int_{S(s)}\right] r^{\gamma_{0}}\left\{|<\tilde{x} \cdot \operatorname{grad} v>|^{2}-\frac{1}{2}\left(|\operatorname{grad} v|^{2}-\tilde{q}|v|^{2}\right)\right\} d S} \\
& \quad+\frac{n-1-\gamma_{0}}{2}\left[\int_{S(t)}-\int_{S(s)}\right] r^{\gamma_{0}-1} \operatorname{Re}[<\tilde{x} \cdot \operatorname{grad} v>\bar{v}] d S \\
& \geq \frac{1}{2} \int_{B(s, t)} r^{\gamma_{0}-1}\left(2 \gamma_{0} \tilde{q}+r<\tilde{x} \cdot \operatorname{grad} \tilde{q}>\right)|v|^{2} d x \\
& \quad+\int_{B(s, t)} 2 \rho^{\prime} r^{\gamma_{0}}|<\tilde{x} \cdot \operatorname{grad} v>|^{2} d x \\
& \quad+\frac{n-1-\gamma_{0}}{2} \int_{B(s, t)}\left(\frac{\gamma_{0}-1}{r}+2 \rho^{\prime}\right) r^{\gamma_{0}-1} \operatorname{Re}[<\tilde{x} \cdot \operatorname{grad} v>\bar{v}] d x .
\end{aligned}
$$

Our aim is to show that any solution $u$ of (1.3) which also satisfies the condition

$$
\liminf _{t \rightarrow \infty} t^{\gamma_{0}} \int_{S(t)}\left\{(1+|q|)|u|^{2}+|<\tilde{x} \cdot \operatorname{grad} u>|^{2}\right\} d S=0
$$

must identically vanish in $\Omega$.

First we put $\rho=0$ in (5.1). Then $v=u, \tilde{q}=q$ and by (A4)' $2 \gamma_{0} q$ 
$+r<\tilde{x} \cdot \operatorname{grad} q>\geq \lambda_{0}$. Thus, by means of (5.2), we can let $t \rightarrow \infty$ to obtain

$$
\begin{aligned}
& \int_{S(s)} r^{\gamma_{0}}\left(|\operatorname{grad} u|^{2}-q|u|^{2}\right) d S \\
& -\left(n-1-\gamma_{0}\right) \int_{S(s)} r^{\gamma_{0}-1} \operatorname{Re}[<\tilde{x} \cdot \operatorname{grad} u>\bar{u}] d S \\
& \geq \lambda_{0} \int_{B(s)} r^{\gamma_{0}-1}|u|^{2} d x \\
& \quad+\left(n-1-\gamma_{0}\right)\left(\gamma_{0}-1\right) \int_{B(s)} r^{\gamma_{0}-2} \operatorname{Re}[<\tilde{x} \cdot \operatorname{grad} u>\bar{u}] d x .
\end{aligned}
$$

Since

$$
\begin{aligned}
& \int_{B(s)} r^{\gamma_{0}-2} \operatorname{Re}[<\tilde{x} \cdot \operatorname{grad} u>\bar{u}] d x \\
& \quad=-\frac{1}{2} \int_{S(s)} r^{\gamma_{0}-2}|u|^{2} d S-\frac{n-3+\gamma_{0}}{2} \int_{B(s)} r^{\gamma_{0}-3}|u|^{2} d x,
\end{aligned}
$$

it follows from (5.3) that

$$
\begin{aligned}
& \int_{S(s)} r^{\gamma_{0}}\left(|\operatorname{grad} u|^{2}-q|u|^{2}\right) d S \\
& -\left(n-1-\gamma_{0}\right) \int_{S(s)} r^{\gamma_{0}-1}\left\{\operatorname{Re}[<\tilde{x} \cdot \operatorname{grad} u>\bar{u}]-\frac{\gamma_{0}-1}{2 r}|u|^{2}\right\} d S \\
& \quad \geq \int_{B(s)} r^{\gamma_{0}-1}\left\{\lambda_{0}-\frac{\left(n-1-\gamma_{0}\right)\left(\gamma_{0}-1\right)\left(n-3+\gamma_{0}\right)}{2 r^{2}}\right\}|u|^{2} d x .
\end{aligned}
$$

This inequality corresponds to (3.5). Hence we see following the line of proof of Lemma 3.1

$$
\int_{B(r o)} r^{m}|u|^{2} d x<\infty \quad \text { for any } \quad m>0
$$

Next we put $\rho(r)=m \log r$ in (5.1). Then by (5.5) and (2.25)

$$
-\int_{S(s)} r^{\gamma_{0}}\left\{2|<\tilde{x} \cdot \operatorname{grad} v>|^{2}-\frac{n-1-\gamma_{0}}{r}|<\tilde{x} \cdot \operatorname{grad} v>\bar{v}|\right\} d S+
$$




$$
\begin{aligned}
& \quad+\int_{S(s)} r^{\gamma_{0}}\left(|\operatorname{grad} v|^{2}-\tilde{q}|v|^{2}\right) d S \\
& \geq \int_{B(s)} r^{\gamma_{0}-1}\left\{\lambda_{0}-2\left(1-\gamma_{0}\right) \frac{m(m-n+2)}{r^{2}}\right\}|v|^{2} d x \\
& \quad+\int_{B(s)} 2 m r^{\gamma_{0}-1}|<\tilde{x} \cdot \operatorname{grad} v>|^{2} d x \\
& \quad+\left(n-1-\gamma_{0}\right) \int_{B(s)} \frac{\gamma_{0}-1+2 m}{r} r^{\gamma_{0}-1} \operatorname{Re}[<\tilde{x} \cdot \operatorname{grad} v>\tilde{v}] d x,
\end{aligned}
$$

where the last term of the right can be estimated from below by

$$
\begin{aligned}
& -\int_{B(s)} \frac{2 m+\gamma_{0}-1}{2} r^{\gamma_{0}-1}|<\tilde{x} \cdot \operatorname{grad} v>|^{2} d x \\
& -\int_{B(s)}\left(n-1-\gamma_{0}\right)^{2} \frac{2 m+\gamma_{0}-1}{2 r^{2}} r^{\gamma_{0}-1}|v|^{2} d x .
\end{aligned}
$$

On the other hand, for $v \in H^{1}\left(B\left(r_{0}\right)\right.$ ) (cf., Lemma 2.5)

$$
\begin{aligned}
& -\int_{S(s)} t^{\gamma_{0}-1}|v|^{2} d S \\
& \geq-\int_{B(s)} r^{\gamma_{0}-1}\left\{|<\tilde{x} \cdot \operatorname{grad} v>|^{2}+\left(1+\frac{n-2-\gamma_{0}}{r}\right)|v|^{2}\right\} d x .
\end{aligned}
$$

Combining (5.6) and (5.7), and choosing $r_{6} \geq r_{0}$ sufficiently large, we have for $s>r_{6}, m>n$ and $0<\alpha \leq \min \left\{\lambda_{0} / 3,1\right\}$

$$
\begin{gathered}
-\int_{S(s)} r^{\gamma_{0}}\left(2|<\tilde{x} \cdot \operatorname{grad} v>|^{2}-\frac{n-1-\gamma_{0}}{r}|<\tilde{x} \cdot \operatorname{grad} v>\bar{v}|\right. \\
\left.+\frac{\alpha}{r}|v|^{2}\right) d S+\int_{S(s)} r^{\gamma^{\prime o}}\left(|\operatorname{grad} v|^{2}-\tilde{q}|v|^{2}\right) d S \\
\geq \int_{B(s)} r^{\gamma_{0}-1}\left\{\frac{\lambda_{0}}{2}-2\left(1-\gamma_{0}\right) \frac{m(m-n+2)}{r^{2}}\right. \\
\left.-\left(n-1-\gamma_{0}\right)^{2} \frac{2 m+\gamma_{0}-1}{2 r^{2}}\right\}|v|^{2} d x,
\end{gathered}
$$

which corresponds to (3.8). Hence we can follow the line of proof of Lemma 3.2 to conclude that for any $k>0$ and $v$ such that $0<v<1$ 


$$
\int_{B\left(r_{0}\right)} e^{k r^{1-v}}|u|^{2} d x<\infty
$$

Finally we return once more to inequality (5.1) with $\rho(r)=k r^{1-\nu}$. Choosing $v$ less than $\gamma_{0}$ and following the line of proof of Theorem 1.1 , we have $u=0$ in $\Omega$. This concludes the proof.

\section{Department of Mathematics,}

Nagoya Institute of Technology

\section{References}

[1] Agmon, S., Lower bounds for solutions of Schrödinger-type equations in unbounded domains, Proceedings of the International Conference of Functional Analysis and Related Topics, Tokyo, 1969, 216-224.

[2] Agmon, S., Lower bounds for solutions of Schrödinger equations, J. d'Anal. Math. 23 (1970), 1-25.

[3] Éidus, D. M., The principle of limit amplitude, Usp. Math. nauk 24 (1969), Vypusk 3 (147), 91-156.

[4] Ikebe, T. and J. Uchiyama, On the asymptotic behavior of eigengunctions of second-order elliptic operators, J. Math. Kyoto Univ. 11-3 (1971), 425448.

[5] Ikebe, T. and T. Kato, Uniqueness of the selfadjoint extension of singular elliptic operators, Arch. Rational Mech. Ann. 9 (1962), 77-92.

[6] Kato, T., Growth properties of solutions of the reduced wave equation with a variable coefficient, Comm. Pure Appl. Math. 12 (1959), 403-425.

[7] Landis, E. M., On some properties of solutions of elliptic equations, Dokl, Akad. Nauk SSSR 107 (1956), 640-643 (Ressian).

[8] Odeh, F., Note on differential operators with purely continuous spectrum, Proc. Amer. Math. Soc. 16 (1965), 363-365.

[9] Protter, M. H., Unique continuation for elliptic equations, Trans. Amer. Math. Soc. 95 (1960), 81-91.

[10] Roze, S. N., On the spectrum of a second-order elliptic operator, Math. Sb. 80 (112) (1969), 195-209 (Russian).

[11] Simon, B., On positive eigenvalues of one-body Schrödinger operators, Comm. Pure Appl. Math. 22 (1967), 531-538.

[12] Weidmann, J., On the continuous spectrum of Schrödinger operators, Comm. Pure Appl. Math. 19 (1966), 107-110.

[13] Weidmann, J., The Virial theorem and its application to spectral theory of Schrödinger operators, Bull. Amer. Math. Soc. 73 (1967), 452-459.

[14] Uchiyama, J., Lower bounds of growth order of solutions of Schrödinger equations with homogeneous potentials, Publ. RIMS, Kyoto Univ. 10 (1975), $425-444$. 